\title{
CRISIS DE LA DEMOCRACIA DE PARTIDOS Y SEGUNDA TRANSICIÓN
}

\author{
JUAN JESÚS GONZÁLEZ
}


SUMARIO

1. VIEJA Y NUEVA POLIÍTICA. 2. LA GENEALOGÍA DE LA CRISIS. 3. EL IMPACTO ELECTORAL DE LA CRISIS: LAS ELECCIONES AUTONÓMICAS Y GENERALES DE 2015. 4. EL NUEVO SISTEMA DE PARTIDOS: DEL PLURALISMO LIMITADO DE LA TRANSICIÓN AL PLURALISMO POLARIZADO. 5. RECAPITULACIÓN. 


\title{
CRISIS DE LA DEMOCRACIA DE PARTIDOS Y SEGUNDA TRANSICIÓN
}

\author{
JUAN JESÚS GONZÁLEZ*
}

Catedrático de Sociología (UNED)

\section{VIEJA Y NUEVA POLÍTICA}

A fin de comprender el tránsito y la tensión entre vieja y nueva política, vamos a comenzar este apartado recordando los postulados del politólogo francés Bernard Manin ${ }^{1}$ sobre la evolución de la representación democrática en las democracias contemporáneas. De acuerdo con este autor, la representación democrática estaría experimentando una transición entre dos tipos ideales que designa como «democracia de partidos» $\mathrm{y}$ «democracia de audiencia». Desde esta perspectiva, podemos interpretar la crisis actual de la democracia española como una crisis de la «democracia de partidos» instituida al amparo del orden constitucional de 1978. Conviene recordar en este punto que dicho orden tenía como objetivo estratégico la conformación de un sistema de partidos fuerte y estable, a fin de evitar y erradicar los problemas de fragmentación e inestabilidad que asolaron la política de los años treinta. En la práctica, estos partidos han acabado funcionando como partidos omnímodos y exentos de control, cada vez más desconectados de la sociedad civil, debido a su arraigada tendencia a la endogamia y la cooptación. Cabría decir, en último término, que los partidos del bipartidismo se han convertido en una partidocracia genuina, entendiendo por tal un sistema político en el que los partidos no buscan tanto representar la sociedad civil como

* Catedrático de Sociología. Facultad de Ciencias Políticas y Sociología. Universidad Nacional de Educación a Distancia. Obispo Trejo, 2. 28040 Madrid. Email: jgonzalez@poli.uned.es

1 Manin, Bernard (1998): Los principios del gobierno representativo, Madrid: Alianza Editorial, Ciencias Sociales. 
colonizarla, llevando a sus últimas consecuencias el conocido proceso de cartelización estudiado por Mair y $\mathrm{Katz}^{2}$.

En condiciones normales, la cartelización de los partidos se ha producido a partir de modelos previos de partido, tales como el partido de masas, los cuales se han ido convirtiendo paulatinamente en partidos de cuadros que sacrificaban la militancia a cambio de nuevos mecanismos de funcionamiento, principalmente financiación pública derivada de su actividad institucional. Ahora bien, debido a las peculiaridades históricas del caso español, los partidos políticos pasaron directamente del (anti)franquismo o, si se quiere, de la clandestinidad a convertirse en agencias paraestatales orientadas no solo a la ocupación de cargos públicos, sino también a la captura de rentas en todos aquellos ámbitos de la vida pública susceptibles de intervención o regulación. En este contexto, la capacidad de los partidos para imponer listas electorales cerradas y bloqueadas, así como la generosidad de las fuentes de financiación pública, unidas a la discrecionalidad en el uso de los recursos públicos y a la ausencia de mecanismos de control verdaderamente eficaces (agencias independientes en materia de control de cuentas, ya sean las cuentas internas de cada partido, ya las cuentas del gasto público...), han dado paso a un proceso de colonización y esquilmo de los innumerables órganos de representación a los que han tenido acceso, ya sean estos el Consejo General del Poder Judicial o los órganos rectores de las Cajas de Ahorro, por no hablar de las agencias que en teoría deberían haber servido para fiscalizar todo este proceso (Consejo de RTVE, Tribunal de Cuentas, etc.).

De acuerdo con la propuesta de Manin, la transición entre una democracia de partidos y una democracia de audiencia estaría afectando, entre otras, a dos dimensiones del sistema democrático:

- La elección y el grado de autonomía de los representantes: mientras en la democracia de partidos los representantes se reclutan entre los activistas y los funcionarios del partido, la democracia de audiencia prima la relevancia mediática de los candidatos, dando protagonismo a los expertos en medios y a los candidatos de mayor habilidad mediática. En la democracia de partidos, el representante es un simple delegado del partido, el cual ocupa un lugar central y mediador entre representantes y representados, en tanto que la democracia de audiencia pone en primer plano al representante, el cual se vale de la citada

2 MaIr, Peter (2015): Gobernando el vacío. La banalización de la democracia occidental, Madrid: Alianza editorial, p. 94. «Con la aparición del partido cártel, los objetivos de la política se vuelven autorreferentes, profesionales y tecnocráticos, y lo que queda de la competición sustantiva entre partidos se refiere menos a diferencias políticas y más —en un sentido congruente con la noción de «democracia de audiencia» de Manin—a la provisión de espectáculo, imagen y teatro» (íbid.). 
relación de confianza personal con los representados para ganar autonomía personal respecto al partido ${ }^{3}$.

- El patrón de opinión pública: mientras en la primera «todas las expresiones de la opinión pública están estructuradas siguiendo divisiones partidistas», como lo prueba la existencia de asociaciones partidarias, prensa de partido, etc. ${ }^{4}$, en la segunda «los canales de comunicación son en su mayor parte políticamente no partidistas», lo que propicia el desalineamiento político de los medios, así como una cierta autonomía entre las preferencias de la audiencia y las preferencias electorales.

Por lo que se refiere a los criterios de elección de los representantes, nos encontramos, en definitiva, con un desplazamiento desde una democracia centrada en los partidos a una democracia centrada en los medios, dentro del cual las elecciones primarias juegan un papel decisivo, por cuanto implican un desplazamiento del ámbito de elección de los representantes desde la cúpula de los partidos a la esfera pública, bajo la vigilancia atenta de los medios, que conseguirían de este modo convertir los procesos de decisión interna de los partidos en debates presididos por la lógica mediática. No es difícil, por tanto, advertir los riesgos de un cambio de este tipo, desde el momento en que los medios pueden estar tentados de presentar las primarias más como un problema de división en el seno de los partidos que como una oportunidad para la democracia interna, riesgo que puede multiplicarse si la decisión de celebrar elecciones primarias no es una decisión unánime de los partidos (tal como ocurriría si estuvieran amparadas por una Ley de Partidos), sino una decisión de alguno de ellos.

En cuanto a la segunda dimensión (el patrón de opinión pública), estaría evolucionando desde unas coordenadas partidistas hacia la configuración — sin que Manin utilice el término_ - de una esfera pública relativamente autónoma de los partidos. La cuestión es tanto más importante por cuanto no se trata solo de un ideal normativo, sino de una tendencia constatable que dio un salto decisivo con el Estatuto de RTVE aprobado en 2006, que consiguió despolitizar el ente radio-televisivo, dignificar su carácter de servicio público y liberarlo de las servidumbre de una televisión comercial mediante la supresión de la publicidad. Como es bien sabido, la llegada del PP al gobierno supuso el retorno a la gubernamentalización del ente público de RTVE, dando al traste con uno de los avances más significativos en la dirección de una democracia de audiencia.

3 En la misma línea de argumentación, otros autores señalan un desplazamiento del proceso de toma de decisiones desde los órganos representativos de los partidos al círculo restringido de asesores de imagen que rodean al candidato (Meyer 2002: 63).

4 Manin, Bernard (1998): Los principios del gobierno representativo, Madrid: Alianza Editorial, Ciencias Sociales, p. 263. 
Es evidente que estos problemas que estamos señalando no agotan ni mucho menos el repertorio temático de la crisis del bipartidismo, en el cual se incluyen también la discusión sobre la reforma del sistema electoral, a fin de hacerlo más proporcional y más representativo, así como la reforma de las agencias públicas de regulación y control, a fin, a su vez, de hacerlas verdaderamente eficaces e independientes. Todo ello se encuadra en el marco más general de una mejora sustancial de la calidad de la democracia española. Desde esta perspectiva, es verdad que los intentos por mejorar dicha calidad no empezaron con los nuevos partidos (de hecho, el PSOE fue pionero tanto en la celebración de primarias como en la des-gubernamentalización de la radio-televisión pública), pero no es menos cierto que la irrupción de los nuevos partidos va asociada a muchos de estos debates y que su eventual consolidación partidaria va a depender de que dichos debates se materialicen en reformas plausibles.

Por último, hay una dimensión de la vieja política que los nuevos partidos vienen también a poner en cuestión, como es el uso del eje ideológico izquierdaderecha como principio estructurante de la competición política. Esto es claro en el caso de Podemos, por cuanto forma parte de su misma estrategia de competición huir del citado eje y competir en el eje que contrapone el establishment de la partidocracia, más conocido como «la casta», al pueblo llano o la gente corriente. Pero lo es también en el caso de Ciudadanos, que presume de ser inclasificable a estos efectos, toda vez que intenta combinar propuestas liberalconservadoras en el campo de la economía con propuestas socialdemócratas en el campo de las políticas sociales.

\section{LA GENEALOGÍA DE LA CRISIS}

Para entender el cambio registrado en el sistema de partidos es preciso comprender la génesis y las principales consecuencias de la crisis económica, política e institucional que se desata en el marco de la Gran Recesión, prestando atención, en particular, a los siguientes factores que intervienen en ella:

- La magnitud de la crisis económica: la explosión del paro y el aumento del precariado.

- La incapacidad de la clase política para dar respuesta a la crisis.

- Las políticas de austeridad y el empobrecimiento de las clases asalariadas.

- La irrupción del 15-M en cuanto movimiento de protesta social y la consiguiente apertura de un nuevo nicho político y electoral.

Como se recordará, la crisis financiera que estalla en 2008 se convierte dos años más tarde en una crisis fiscal, como consecuencia de que, por un lado, los 
ingresos fiscales asociados a la burbuja inmobiliaria se evaporan, en tanto que, por otro, las exigencias de gasto público derivadas de la crisis se disparan. Con esta premisa inicial, en 2010 irrumpen en España las políticas de austeridad con las que la Unión Europea pretendía atajar la crisis de deuda soberana que se extendía por toda la periferia de la eurozona, dando lugar a una situación generalizada de depresión económica y deflación.

En términos de riqueza, el PIB, que había sufrido un bache traumático en 2009, con una caída del 4\%, recayó nuevamente en 2012, con una caída adicional del $2 \%$, que traducido a renta disponible, hizo que los hogares perdieran en los seis años de recesión (2008-2013) toda la ganancia acumulada en la década anterior. Más dramática aún fue la evolución en términos de paro, por cuanto la tasa se disparó desde el $8 \%$ al $26 \%$ de la población activa, un salto sin precedentes, que arrastró no solo un aumento desorbitado del paro, sino un aumento paralelo del precariado, entendiendo por tal el segmento laboral que se mueve entre la temporalidad y el desempleo. Y así como en tiempos de bonanza la temporalidad podía interpretarse como un coste en el proceso de inserción laboral (encarnado entonces en la figura del mileurista), el precariado que surge de la recesión tiene un significado distinto, pues representa la cronificación de un segmento laboral que ve bloqueadas de manera indefinida sus expectativas de estabilización y promoción laboral. Esta nueva situación abre un debate social sobre la eventualidad de una generación perdida de jóvenes precarios que encuentran en la emigración la única vía de escapar a la radical imposibilidad de poner en práctica sus proyectos personales y familiares. Nada tiene de extraño, por tanto, que el clivaje generacional se convierta, a partir de ese momento, en una variable fundamental para analizar el desarrollo de los acontecimientos, tanto los relativos a la irrupción del 15-M como a su posterior traducción política (Podemos).

El segundo factor a tener en cuenta es la incapacidad de la clase política para encontrar una respuesta, si no satisfactoria, sí, cuando menos, consensuada a la crisis económica. Siguiendo con la dinámica previa de polarización política que había caracterizado la década anterior, las políticas anti-crisis pasaron de la inacción de los primeros años de la recesión (2008-2009) al frenesí austeritario de los primeros años de legislatura popular (2012-2013), ambos bienios caracterizados por la más completa ausencia de acuerdo y colaboración entre los dos grandes partidos. Es claro que, entre una opción y otra, había múltiples vías para evitar el nivel de rechazo social de las fórmulas elegidas a partir de 2010, pero eso hubiese requerido un tipo de colaboración política al que las elites del bipartidismo no estaban acostumbradas. Dadas las políticas de descrédito mutuo, la pérdida de legitimidad terminó afectando por igual a izquierda y derecha, a diferencia de lo ocurrido en los años ochenta cuando una vía alternativa de sali- 
da de la crisis (una política de ajuste económico compensada mediante políticas sociales) dio lugar a enfrentamientos entre el gobierno y los sindicatos pero en ningún momento se tradujo en crisis política ni, mucho menos, en una mutación del sistema de partidos.

En definitiva, la política de devaluación interna practicada por el gobierno popular en el bienio 2012-2013 ha tenido como principales objetivos la reducción de costes salariales (reforma laboral de 2012) y el recorte de las políticas sociales, lo que, unido al efecto devastador de la recesión, completa así el proceso de empobrecimiento de las clases asalariadas y lleva la economía al terreno de la deflación. Pues en su intento por reajustar los salarios a los niveles de productividad que se perdieron en tiempos de la burbuja, la austeridad auto infligida ha servido, por el momento, para contraer la demanda interna y agrandar la distancia salarial entre los trabajadores precarios y el resto, con especial incidencia entre los jóvenes.

Hay que tener en cuenta, por otro lado, que en el terreno de la opinión pública la clase política se convierte, a partir de 2010, en uno de los problemas más acuciantes de la agenda pública, el cual se combina, a partir de 2012, con la corrupción y el fraude, de manera que a la preocupación inicial por la incapacidad de la clase política para enfrentarse a la crisis se suma, más tarde, la preocupación por la financiación de los partidos y su funcionamiento interno. En su efecto combinado, el triángulo temático compuesto por un paro masivo, una clase política incompetente y una percepción de corrupción generalizada sienta las bases de una puesta en cuestión del orden institucional en su conjunto y prepara el camino para un ciclo de protesta social que tiene como protagonista, primero, al movimiento 15-M y, más tarde, al partido-movimiento Podemos (que también podría caracterizarse como ciberpartido, dada su dependencia de las redes sociales que se tejen con la extensión de la protesta).

Llegados a este punto, conviene que nos detengamos por un momento en la secuencia de los acontecimientos: para empezar, mayo de 2010 pone fin de manera abrupta a la estrategia socialista de contención de la crisis que en otro lugar hemos caracterizado como «salida social de la crisis» ${ }^{5}$, mediante la cual el expresidente Zapatero intentó evitar que los costes de la misma recayesen sobre aquellos sectores sociales que no habían tenido responsabilidad alguna en su origen. Es el momento en que la célebre Troika y sus hombres de negro ponen en marcha el primer rescate de la economía griega, un rescate que casi todos los implicados (Comisión Europea, FMI...) han terminado por reconocer como descabe-

5 González, J. J. y P. Chavero (2012): «Política en portada: la construcción mediática de la crisis», en Colino y Cotarelo: España en crisis. Balance de la segunda legislatura de Rodríguez Zapatero, Valencia: Tirant Humanidades: 290-310. 
llado y contraproducente, no solo por lo desproporcionado de sus medidas sino también por la fuerte resistencia social que generó. En España, Zapatero anuncia el primer paquete de recortes (empezando por un recorte del $5 \%$ en el sueldo de los funcionarios), rápidamente contestado por una huelga general que pone fin a la laboriosa paz social que había presidido su mandato desde 2004. A partir de ese momento, los sondeos comienzan a registrar un auténtico desplome en las tasas de fidelidad de los votantes socialistas.

Tras la huelga, el gobierno y los sindicatos hicieron un último intento por recomponer la paz social mediante un acuerdo de reforma del sistema de pensiones, pero el repentino frenesí reformista que se apoderó de Zapatero («cueste lo que me cueste») ya no pudo impedir la recaída de la economía que se registra en 2011, colocando de esta manera al gobierno socialista ante una inquietante expectativa electoral.

En vísperas de la celebración de las elecciones municipales y autonómicas de 2011, se produjo la ocupación de un espacio público emblemático como es la madrileña Puerta del Sol por parte de «los indignados», que ponen en circulación los eslóganes de la protesta («no nos representan», «lo llaman democracia y no lo es»...), al tiempo que formulan sus principales reivindicaciones: reforma de la ley electoral, ley de partidos, denuncia de la corrupción, vivienda digna, etc. Es claro que el contenido y el tono de la protesta estaban en línea, sobre todo, con la estrategia de IU, que era en ese momento la organización política mejor situada para hacerse cargo de las citadas reivindicaciones. Ahora bien, la situación económica del país propició un voto masivo de castigo a los socialistas que permitió al PP conseguir una mayoría sin precedentes, la cual preparó el camino para la mayoría absoluta de este mismo partido en las elecciones generales de noviembre.

Por su parte, el partido socialista se despidió del gobierno con una medida tan controvertida como fue la reforma del artículo 135 de la CE, en el marco de las exigencias de estabilidad presupuestaria planteadas por las instituciones europeas a iniciativa de Alemania. En principio, cabe discutir si la estabilidad presupuestaria y la contención del déficit deben formar parte de la normativa constitucional. El problema es que una iniciativa de este tipo, pactada con el PP en vísperas de su llegada al gobierno, se interpretó como un cheque en blanco a favor de los recortes y como una forma de anteponer el servicio de la deuda a las políticas sociales. Cabría decir, por tanto, que la reforma del artículo 135 de la CE sirvió, en último término, para visualizar, a ojos de la opinión pública, la ruptura del pacto social que se había inaugurado con los Pactos de la Moncloa (antecedente inmediato del gran pacto constitucional de 1978).

Conviene recordar, en este punto, que dicho pacto gravitaba sobre la defensa de los tres pilares en los que se ha basado el estado de bienestar en España: educa- 
ción, sanidad y pensiones. Y conviene recordar también que así como las pensiones son competencia de la Administración General del Estado, las partidas de educación y sanidad son competencia autonómica, lo que ha permitido al gobierno del PP descargar la mayor parte de los recortes sobre las espaldas de las CC.AA. De ahí que mientras el gasto en protección social (pensiones principalmente) ha seguido aumentando en términos de porcentaje sobre PIB (pasando del 16\% al 17,6\% entre 2009 y 2013), el gasto en educación y sanidad ha pasado del 11,4\% al $10 \%$ en ese mismo periodo, lo que representa una caída superior al 10\% (Fundación BBVA-Ivie 2015). De esta forma, el Estado de bienestar español agudiza una de sus características más genuinas, como es el sesgo a favor de las generaciones mayores (pensiones) y en contra de los jóvenes (educación). A lo largo de ese tiempo, las distintas mareas surgidas en defensa de los servicios públicos (la marea verde de la educación y la marea blanca de la sanidad) han constituido un baluarte en la lucha contra la austeridad y los recortes, convirtiendo el Estado de bienestar en uno de los escenarios cruciales de la protesta.

Aunque no es fácil evaluar el impacto del 15-M en las elecciones de 2011, podemos identificar dos vectores principales: un refuerzo muy considerable del voto a IU, que pasa del 3,8\% al 6,9\% del voto válido (y lo que es más importante: de 2 a 11 diputados), junto a un desplome sin precedentes del PSOE, que pasa del $43,9 \%$ al 28,8\%, con la consiguiente pérdida de 59 diputados nacionales. Ahora bien, si por algo se caracterizan las elecciones de 2011, en comparación con las de 2008, es que así como estas registraron la tasa más alta de participación de todas las elecciones de continuidad celebradas en España desde 1979 (75\%), en 2011 se registró la tasa más baja de participación de todas las elecciones de cambio (69\%), lo cual se explica en primer lugar por la deserción masiva de los votantes socialistas, que se refugiaron en la abstención. Este dato nos pone en la pista de una bolsa de abstención muy poblada que se forma en el lado izquierdo del espectro ideológico, cuyo epicentro podemos localizar en la posición 3 de la escala ideológica, que ha sido, sucesivamente, el punto medio de auto ubicación de los simpatizantes del 15-M, el punto de fuga de los votantes socialistas en 2011 y el epicentro del electorado de Podemos en el momento de su irrupción.

Al término de este repaso por los cambios y acontecimientos más reseñables de la crisis, nos encontramos con algunos de los principales factores coadyuvantes en el proceso de reconfiguración del sistema de partidos. Por lo pronto, las transformaciones estructurales derivadas de la gran recesión y, en particular, el surgimiento de un precariado de una magnitud sin precedentes, el cual lleva asociada la conformación de amplios sectores sociales que no se sienten representados por los partidos tradicionales y se quedan al margen del proceso político. Es tarea, por tanto, de los partidos emergentes establecer una nueva agenda 
política capaz de atender los intereses y las demandas de estos sectores sociales a fin de reincorporarlos al sistema político. El segundo factor es la percepción de convergencia y redundancia en la actuación de los partidos tradicionales, toda vez que sus políticas parecen obedecer al principio de que «no hay alternativa», lo que invalida y deslegitima el proceso de debate público y de confrontación democrática. A todo lo cual podemos añadir un tercer factor, como es la pérdida de eficacia de las redes clientelares urdidas por los partidos tradicionales, que entran en proceso de descomposición como consecuencia, por un lado, del estallido de la burbuja y de la consiguiente reducción de las rentas a repartir y, por otro, de los escándalos de corrupción que han asolado la opinión pública española durante los últimos años y que han servido para poner a la luz extensas zonas de penumbra que se habían instalado en la política española.

\section{EL IMPACTO ELECTORAL DE LA CRISIS: LAS ELECCIONES AUTONÓMICAS Y GENERALES DE 2015}

Con estas premisas, en las elecciones autonómicas de 2015 que se celebraron en trece CC.AA. más Andalucía, el bipartidismo retrocedió 18 puntos porcentuales y aunque el ganador de aquellas elecciones tomadas en su conjunto fue el PP $(29,7 \%)$, el gran beneficiario fue el PSOE, que con el 27,5\% de los votos consiguió entrar a formar parte de nueve gobiernos regionales (por su parte, el PP perdió seis y retuvo cuatro). Por lo que se refiere a los partidos emergentes, el resultado de Ciudadanos en las elecciones autonómicas de 2015 no fue tan espectacular como el de Podemos en términos cuantitativos, pues así como Podemos obtuvo el 14,4\% del voto en las 14 CC.AA. en las que hubo elecciones, Ciudadanos se hubo de conformar con el $10 \%$. Ahora bien, Ciudadanos fue decisivo en la formación de seis gobiernos regionales (entre ellos Andalucía y Madrid, las comunidades más grandes), con la ventaja estratégica de poder pactar con dos partidos (PP y PSOE).

En consecuencia, las elecciones autonómicas de 2015 sirvieron para poner en marcha un nuevo sistema de partidos organizado en torno a un doble eje de competición: el eje ideológico que contrapone izquierda y derecha (en el que se había movido el bipartidismo hasta ese momento) y el eje que contrapone vieja y nueva política. De tal suerte que los partidos ya no competían en un único eje, sino que competían en torno a una diagonal que tenía en un extremo al PP (el más escorado a la derecha y el más identificado con la vieja política) y en el otro a Podemos (el más escorado a la izquierda y el mejor exponente de la nueva política —al menos el más exigente en materia de ruptura institucional). Así las cosas, aquellas elecciones arrojaron un doble saldo: por un lado, una situación de equilibrio entre dos 
bloques ideológicos (PP-C's y PSOE-Podemos), cada uno de los cuales reunía en torno a un $40 \%$ del voto total. Por otro, una situación de desequilibrio entre vieja y nueva política a favor de la primera (el bipartidismo consiguió el $57 \%$ del voto, frente a un $25 \%$ de los emergentes), lo cual no impidió que la nueva política fuera decisiva en la mayoría de los acuerdos de investidura que se firmaron. De hecho, Podemos participó en siete y Ciudadanos en cinco de los catorce acuerdos de investidura firmados entonces (en todos, menos Asturias y Canarias).

Todo ello preparó el camino de Ciudadanos para convertirse en la segunda fuerza parlamentaria en Cataluña (muy por delante del PSOE y del PP) en las elecciones catalanas de septiembre de ese mismo año, en las que las candidaturas nacionalistas se agruparon ( «Junts pel Sí») con el fin de convertir dichas elecciones autonómicas en un plebiscito sobre la independencia de Cataluña. En consecuencia, Ciudadanos se convirtió en la primera fuerza capaz de hacer frente al independentismo, al tiempo que la mejor situada para capitalizar el descontento y la conmoción que la intentona separatista produjo en el resto de España, donde, si bien hasta ese momento Ciudadanos había crecido a costa del PP, a partir de entonces lo podía hacer también a costa del PSOE, dando prueba así de que su dinámica de crecimiento no estaba subordinada al eje izquierda-derecha. De hecho, bastaba con que el tema catalán pasase al primer plano de la agenda política para que Ciudadanos creciera indistintamente a uno y otro lado del espectro ideológico, cerrando así el círculo que se había iniciado en sus mismos orígenes, cuando la pulsión nacionalista de Maragall les había abierto el camino de su implantación en Cataluña. Y en la medida en que el binomio "paro-corrupción» (que había acaparado la agenda de las elecciones municipales y autonómicas) cedió terreno a la amenaza separatista derivada de las elecciones catalanas, la nueva agenda temática (unidad nacional, defensa de la Constitución, etc.) resultó mucho más propicia para Ciudadanos, que se encontró con la posibilidad de competir con el PSOE por el segundo puesto en toda España, debido a las ambigüedades del PSOE con el problema catalán.

Llegamos así a las elecciones generales de 2015. Para su mejor comprensión, vamos a tomar como punto de partida la encuesta pre-electoral del CIS realizada con más de un mes de antelación respecto a la fecha de las elecciones. Esta encuesta nos proporciona, por tanto, la correlación de fuerzas existente tras las elecciones catalanas de septiembre, una coyuntura bien distinta del clima de opinión que había caracterizado las elecciones autonómicas de primavera a las que ya hemos hecho referencia. Pues así como estas últimas elecciones respondían a un escenario de equilibrio entre bloques ideológicos, la victoria de «Junts pel Sí» en las elecciones catalanas y la consiguiente amenaza secesionista dieron lugar 
a un escenario típico de cierre de filas en torno a la bandera en el que las fuerzas a la derecha del espectro ideológico jugaban con ventaja.

Si observamos los datos de la mencionada encuesta pre-electoral del CIS, nos encontramos con una situación de doble empate entre viejos y nuevos partidos: empate técnico entre PP y PSOE, por un lado, y C's y Podemos, por otro. En cuanto al empate entre PP y PSOE, conviene tener en cuenta, no obstante, que si atendemos al dato de recuerdo de voto, nos encontramos con un problema de infra-representación del electorado popular que se interpreta por lo general como una situación de voto oculto a favor del PP. De ahí que la estimación de voto a este partido estuviese muy por encima del indicador de voto+simpatía (nada menos que diez puntos por encima), pero muy cerca del resultado realmente obtenido por el PP en las elecciones, de manera que la campaña no pareció influir en los resultados de este partido. Por su parte, el PSOE se encontraba en la situación opuesta al PP: su electorado estaba sobre-representado en la encuesta, lo que explica que el CIS optase por una estimación conservadora que quedó ligeramente superada el día de las elecciones. Hasta aquí no hubo mayores sorpresas.

Las sorpresas estaban reservadas para los nuevos partidos y se produjeron por partida doble. Dejando aparte el problema de que los nuevos partidos carecían del dato de recuerdo de voto por cuanto era la primera vez que se presentaban a elecciones de este tipo, el principal problema estaba centrado en la asignación de una bolsa importante de votantes indecisos que dudaban entre varias opciones. Toda vez que en su mayor parte dudaban entre C's y otro partido, los analistas entendieron que C's era el partido con mayor potencial de crecimiento por lo que le asignaron la estimación más generosa. Sin embargo, C’s no respondió en modo alguno a estas expectativas. Más bien al contrario, C’s se encontró, por lo pronto, con que el tema catalán se desactivó durante la campaña (dado el impasse producido por la negativa de la CUP a investir a Artur Mas), al tiempo que realizaba una campaña manifiestamente mejorable que culminó en su recta final con el anuncio de que se abstendría para facilitar la investidura del partido más votado, renunciando así a la posibilidad de actuar como partido bisagra y cortando, por tanto, el flujo de antiguos votantes socialistas que estaban dudando entre C's y una opción de izquierda. En consecuencia, C's perdió toda posibilidad de crecimiento respecto a la intención de voto manifestada durante la precampaña en la encuesta del CIS, al contrario que Podemos y sus operaciones de confluencia, que aprovecharon todas las posibilidades que les ofrecía la campaña para superar de lejos las previsiones de los analistas.

Pues una vez desactivado el tema catalán, la campaña de las generales volvió a los cauces por los que había discurrido la campaña de las autonómicas celebradas en primavera. Frente a un gobierno que trataba de capitalizar la recuperación eco- 
nómica y un público escéptico que no la percibía, los partidos de oposición apelaron a los temas que habían dominado la agenda (paro, problemas económicos, corrupción...) para neutralizar el discurso gubernamental. En ese marco conceptual, nadie mejor que Podemos para apelar a la memoria de lo que había sido la segunda legislatura de Rodríguez Zapatero y la primera de Rajoy y trasladar a la opinión pública el convencimiento de que, tras la ruptura del pacto social, una segunda transición estaba en marcha. De ahí su resultado espectacular (20,7\%), duplicando los mejores registros obtenidos por la izquierda no socialista: el 10,8\% obtenido por el PCE en 1979 y el 10,5\% obtenido por IU en 1996.

Quizá el dato que mejor resume el éxito de la campaña de Podemos fuera el resultado de los debates electorales de nuevo formato que proliferaron en 2015 y que, a diferencia de todos los celebrados con anterioridad, dejaron de estar sujetos a las reglas acordadas entre las cúpulas de los partidos y pasaron a ser debates guiados por criterios puramente periodísticos. Desde este punto de vista, particular repercusión tuvo el debate a cuatro promovido por Atresmedia y retransmitido por Antena 3 y La Sexta, más conocido como el «debate decisivo», que consiguió igualar la audiencia del debate a dos organizado por la Academia de la televisión ( $48 \%$ de share), el cual siguió la pauta de ocasiones anteriores. Pues bien, de la decena larga de encuestas que preguntaron a los espectadores quién consideraban el ganador del «debate decisivo», todas ellas sin excepción dieron como ganador a Pablo Iglesias. En promedio, nada menos que el 59\% de los entrevistados así lo expresaron, lo que parece certificar que la llamada de Pablo Iglesias, en el último minuto del «debate decisivo», a mantener viva la llama de la indignación que había prendido el 15-M ahondó la grieta electoral en el seno de la izquierda, de tal suerte que la «mayoría social de progreso» en la que se había asentado la gobernabilidad socialista durante décadas pasaba a convertirse en un espacio sociopolítico bifronte, toda vez que los once millones de votos conseguidos por el PSOE en 2008 aparecían, siete años más tarde, repartidos a partes casi iguales entre el campo socialista y la nueva izquierda.

Por si el dato no fuera suficientemente elocuente, conviene advertir que esta división no refleja solo la profunda fractura social originada por la crisis, sino que refleja también una segunda línea divisoria relativa al modelo de organización territorial que enfrenta la tradicional concepción federal de la familia socialista con la nueva concepción confederal de la nueva izquierda, para la cual la soberanía nacional deja de ser una soberanía compartida y pasa a ser la soberanía de cada uno de los pueblos que quieran libremente adherirse al proyecto de una España reconstituida de acuerdo a nuevos parámetros. Recordemos, en este punto, que las operaciones de confluencia negociadas por Podemos en Galicia, Cata- 
luña y la Comunidad Valenciana aportaron nada menos que ocho de los 20,7 puntos porcentuales conseguidos por la coalición el 20-D.

Este éxito electoral sin precedentes de Podemos ilustra mejor que nada las consecuencias últimas de aquella dinámica endiablada en virtud de la cual la explosión de la burbuja financiera y el consiguiente derrumbamiento del «ladrillo» (en cuanto modelo productivo característico de la pasada década) dio paso a una crisis social de largo alcance que, lejos de encontrar la respuesta política adecuada, transmutó en una crisis institucional sin precedentes, llevándose por delante el bipartidismo como sistema de gobierno y contribuyendo así a una profunda reorganización del mapa político. Ahora bien, para alcanzar tal éxito fue necesaria la aparición de un puñado de emprendedores políticos que valiéndose de un conocimiento exhaustivo de los resortes comunicativos que operan en las democracias mediáticas, así como de una astucia organizativa más que probada, consiguieron sentar las bases de una nueva estructura partidaria que todavía resulta difícil de clasificar, a medio camino entre el ciberpartido y el partido-movimiento.

Al término de este proceso de convulsión socioeconómica seguida de crisis institucional y de mutación del sistema de partidos, este sistema vuelve a un nuevo estadio de pluralismo que recuerda en cierto modo al sistema inicial, cuando las elecciones inaugurales de la transición (1977) dieron paso a un sistema de cuatro partidos. Conviene recordar, no obstante, que en aquella ocasión los electores premiaron a los partidos más centrados tanto a un lado como al otro del espectro ideológico, dando el mayor protagonismo a UCD (que dominaba el centro-derecha) y al PSOE (que dominaba el centro-izquierda), por cuanto ambos partidos representaban mejor el deseo de romper con el pasado que animaba el ánimo de los españoles en aquel momento. Por contraste, tanto la Alianza Popular de Manuel Fraga como el PCE de Santiago Carrillo aparecían asociados al pasado, tanto si se trataba del pasado inmediato del franquismo como del pasado remoto de la guerra civil ${ }^{6}$ — de ahí el papel secundario que les correspondió en aquel momento histórico ${ }^{7}$.

${ }^{6}$ A diferencia del PSOE, el PCE de Santiago Carrillo no había hecho un congreso de relevo generacional y de ruptura con el pasado como el que había celebrado el PSOE en Suresnes (1974), que dio paso a la generación de Felipe González.

7 Hay otra manera, no obstante, de medir la correlación de fuerzas en relación con la ruptura política que propugnaban las fuerzas de izquierda por oposición a la vía reformista defendida por la derecha. Desde este punto de vista, ambos bloques (AP y UCD, por un lado, frente a PSOE, PSP y PCE, por el otro) quedaron casi empatados en número de votos (no así en escaños, debido a la ley electoral que había sido elaborada por esa misma derecha en 1976, durante la discusión de la Ley para la Reforma Política; de hecho, en 1977 PSOE, PSP y PCE obtuvieron cien mil votos más, en tanto que UCD y AP obtuvieron 39 escaños más. Véase Castro 2008). 
Pues bien, en nuestro caso (2015) ha ocurrido justamente al revés de lo que ocurriera en 1977: los electores han seguido apoyando a los viejos partidos por encima de los nuevos y esto ha ocurrido tanto a la derecha como a la izquierda del espectro ideológico, toda vez que el PP ha conseguido más apoyos que Ciudadanos y que el PSOE, a su vez, ha conseguido más apoyos que Podemos. Este dato es clave para entender el alcance de la llamada «segunda transición» con respecto a la primera y, en particular, para entender por qué el deseo de ruptura con el pasado que anima al electorado en estos momentos es menor que entonces. Ahora bien, la correcta interpretación de esta diferente intensidad en la voluntad de ruptura pasa por tener en cuenta una diferencia fundamental entre la sociedad de los años setenta y la actual, como es el avanzado proceso de envejecimiento demográfico que se ha producido entre medias. Dicho en otras palabras: ¿qué hubiera pasado en 2015 si la sociedad española hubiera tenido la misma estructura de edades que tenía en la segunda mitad de los setenta? Pues que el peso relativo de los viejos y los nuevos partidos estaría mucho más equilibrado, de tal manera que tendríamos no solo una situación de equilibrio entre bloques ideológicos (izquierda-derecha) sino también de equilibrio entre vieja y nueva política.

\section{EL NUEVO SISTEMA DE PARTIDOS: DEL PLURALISMO LIMITADO DE LA TRANSICIÓN AL PLURALISMO POLARIZADO}

La principal innovación de las elecciones de 2015 radica en la conformación de un sistema de partidos que, a diferencia del bipartidismo, donde la competición política estaba organizada a partir de un único eje (izquierda-derecha), la competición partidaria se articula ahora a partir de un doble eje en el que entra en juego la oposición entre vieja y nueva política. Con esta premisa inicial, se abría la posibilidad de un acuerdo transversal entre los nuevos partidos: no hacía falta que alcanzasen un acuerdo de gobierno completo, pero sí que hubiese algún tipo de complicidad en torno a los temas propios de la «nueva política»: lucha contra la corrupción (refuerzo y autonomía del poder judicial), cambio del sistema electoral, regeneración institucional...

Este acuerdo transversal podía dar lugar a una legislatura corta, enfocada en los temas propios de la «nueva política», de manera que los temas de contienda propios del eje ideológico (I-D) quedasen sujetos a una fórmula de compromiso de carácter transitorio. Se trataba, en suma, de dejar en segundo plano las diferencias que pudiera haber en política económica y social con el fin de dar prioridad a una «segunda transición» centrada en la regeneración del sistema democrático. El problema es que, cuando llegó el momento de la negociación, 
Podemos puso como condición al PSOE que Ciudadanos quedase fuera de la misma, lo que fue rechazado por Pedro Sánchez ${ }^{8}$. Y cuando finalmente Alberto Garzón (IU) persuadió a Podemos de que se sentase a negociar, Ciudadanos ya tenía un acuerdo prácticamente cerrado con el PSOE que afectaba a todos los temas de gobierno incluidos aquellos que Podemos no podía aceptar (salvo riesgo de traicionar su propio programa). A partir de ese momento, el PSOE quedaba supeditado a dicho acuerdo.

¿A qué obedece este desinterés de Podemos por la negociación? La explicación habitual es que el objetivo estratégico de Podemos no es tanto desbancar al PP como ocupar el espacio del PSOE. De hecho, los dirigentes de Podemos llegaron al 20-D convencidos de que les había faltado una semana de campaña para derrotar al PSOE, de manera que podemos interpretar todo lo ocurrido después como una continuación de la campaña de Podemos para conseguir dicho objetivo. Pero hay otra manera de explicarlo que pasa por tener en cuenta la dinámica política del bipartidismo a lo largo de los últimos quince años, desde el momento en que el PP consigue su primera mayoría absoluta. Es el momento en que José María Aznar pone en cuestión algunos consensos básicos, tal como ocurrió con la huelga general de 2002 o la guerra de Irak de 2003, lo cual fue seguido de respuestas simétricas como el Pacto del Tinell, que dejaba al PP fuera de cualquier negociación política en Cataluña. Se trata, en cualquier caso, de maniobras de exclusión mutua que pusieron en marcha una dinámica endiablada de polarización que tiene su clímax durante la crisis del 11-M, en medio de la cual Aznar se negó a convocar a la Comisión del Pacto por las libertades y contra el terrorismo. Esa maniobra le costó el gobierno al PP, que perdió las elecciones, pese a lo cual el PP redobló la apuesta, pues, como se recordará, la estrategia culmina con los intentos de Rajoy de deslegitimar al gobierno elegido unos días después, estrategia que marcó la primera legislatura de Zapatero, cuando la crispación llegó a ser comparable a los últimos años del gobierno de Felipe González.

Aquella decisión estratégica de Aznar constituye el mejor ejemplo de que, con llamativa frecuencia, los partidos han preferido atender las demandas de sus propias bases, en vez de competir por el votante medio, como sería de esperar. Más concretamente, lo que este ejemplo sugiere es que los partidos se muestran receptivos a las demandas del votante medio mientras tratan de conseguir su voto, pero se radicalizan una vez que lo consiguen, momento a partir del cual traicionan los intereses de ese mismo votante medio mediante apuestas de alto riesgo electoral. ¿Cómo explicar esta opción estratégica por la polarización? Hay

8 «Sánchez rechaza la "negociación en exclusiva y excluyente" de Iglesias», El Mundo, 5-02-2016.

(C) UNED. Revista de Derecho Politico

N. ${ }^{\circ} 100$, septiembre-diciembre 2017, págs 615-638 
varias razones por las cuales la polarización resulta una opción racional de los partidos, entendiendo por tal una estrategia orientada a achicar el centro mediante incentivos para que sus potenciales habitantes lo abandonen, alineándose con alguno de los frentes en conflicto.

- La primera razón es que la polarización aumenta el sentimiento de inseguridad de los votantes menos ideológicos y más moderados, facilitando el cierre de filas en torno a dos bandos ideológicos que se convierten en trincheras 9 .

- La segunda razón es que la polarización induce respuestas simétricas del adversario político que generan una espiral de amenazas e incertidumbres, lo que refuerza el punto anterior ${ }^{10}$.

- La tercera es el achique del espacio ideológico de centro, como consecuencia del abandono del juego político y del consiguiente refugio en la abstención ${ }^{11}$. La conversión de la política en un deporte agonístico que se agota en sí mismo, pero que no reporta mejora alguna en la convivencia y en la resolución de los problemas, induce sentimientos de rechazo, cuando no de cinismo ( «todos son iguales»), desalentando la participación política de los moderados.

Esta dinámica puede parecer repulsiva o indeseable pero es perfectamente racional $^{12}$, tal como lo prueban los resultados obtenidos por los dos grandes partidos en las elecciones de 2008, en las que consiguen acaparar el $84 \%$ de los votos, un record absoluto. Es probable, por tanto, que los partidos intenten reeditar esta estrategia, tanto más por cuanto los partidos que pueden protagonizarla están cada vez más lejos entre sí, lo que facilita la polarización. Pues a diferencia del pluralismo limitado que caracterizó el sistema de partidos inicial de la transición, el cual estaba sesgado al centro (dada la supremacía de UCD respecto a AP y del PSOE respecto al PCE), el nuevo pluralismo está cada vez más polarizado, dada la supremacía del PP (que se ubica en la casilla 8 de la escala ideológica) respecto a Ciudadanos (que se ubica en la 6), así como la dura pugna por la hegemonía que mantienen Unidos Podemos (casilla 2) y el PSOE (casilla 4).

9 En último término, la polarización pretende sustituir la distribución de las preferencias, que típicamente tiene una distribución normal en torno a un punto medio más o menos central, por una distribución bimodal. Corrales, Javier (2005): «In Search of a Theory of Polarization: Lessons from Venezuela, 1999-2005», Revista Europea de Estudios Latinoamericanos y del Caribe 79, 105-118.

10 Ibid.

11 Ansolabehere, Stephen y Shanto Iyengar (1995): Going Negative. How Political Advertisements Shrink \& Polarize the Electorate, New York: The Free Press.

12 Corrales, Javier (2011): «Why Polarize? Advantages and Disadvantages of a RationalChoice Analysis of Government-Opposition Relations under Hugo Chávez», en Ponniah y Eastwood: The Revolution in Venezuela. Social and Political Change under Chávez, Harvard University Press. 
A fin de tener una cabal comprensión del grado de polarización alcanzado con el sistema de partidos actual, la tabla adjunta aporta tres tipos de datos: a) la auto ubicación ideológica de los votantes; b) la ubicación ideológica de los partidos según los votantes; y c) el porcentaje de voto obtenido por cada uno de los partidos. La tabla permite, por otra parte, comparar el sistema de partidos de la transición y el sistema de partidos actual. Tal como se puede observar en ella, la distancia entre los votantes de los partidos más alejados es ahora inferior de lo que era entonces (habiéndose reducido de 4,8 puntos en 1980 a 4 puntos en 2015), al tiempo que la distancia entre esos mismos partidos que constituyen los polos ideológicos del sistema ha aumentado desde los 5,6 puntos, en el caso de la transición, hasta los 5,9 puntos actuales. Ahora bien, el principal factor de polarización no radica tanto ahí como en la distribución del voto, por cuanto los polos ideológicos del sistema (PCE y AP) representaban el 17,7\% del voto en 1977, en tanto que los polos actuales (PP y Podemos + IU) representaron nada menos que el $53 \%$ del voto registrado en las elecciones de 2015.

Ubicación ideológica de los votantes y de los partidos (1980-2015)

\begin{tabular}{|c|c|c|c|c|c|}
\hline & $\begin{array}{c}\text { PCE/ } \\
\text { Pod's+IU }\end{array}$ & PSOE & $\begin{array}{c}\text { UCD/ } \\
\text { C's }\end{array}$ & $\mathrm{AP} / \mathrm{PP}$ & $\begin{array}{c}\text { Votante } \\
\text { medio }\end{array}$ \\
\hline 1980 Votantes & 2,5 & 3,6 & 5,6 & 7,3 & 4,5 \\
\hline 1980 Partidos & 2,3 & 3,4 & 5,9 & 7,9 & \multirow{4}{*}{4,7} \\
\hline \% de voto 1977 & 9,3 & 29,4 & 34,8 & 8,4 & \\
\hline 2015 Votantes & 3,0 & 3,7 & 5,5 & 7,0 & \\
\hline 2015 Partidos & 2,2 & 4,4 & 6,6 & 8,3 & \\
\hline$\%$ de voto 2015 & 24,3 & 22,0 & 13,9 & 28,7 & \\
\hline
\end{tabular}

Fuente: Maravall 1984: 45-47 y Cis 3126 (pos-electoral 2015).

Esta nueva correlación de fuerzas plantea un riesgo cierto de que el actual pluralismo entre en una dinámica bien distinta de la que siguió el pluralismo inicial, la cual fue una dinámica orientada a la conquista del centro. Caben, en este sentido, dos posibilidades según que las estrategias de negociación que han puesto en marcha los partidos bisagra (PSOE y Ciudadanos) den lugar a fórmulas de compromiso que sean factibles en términos de gobernabilidad o, por el contrario, entremos en una dinámica de polarización y de frentismo que termine por beneficiar a las posiciones más radicales. Que la democracia española entre en una u otra dinámica es una cuestión que tiene que ver, por lo pronto, con la 
capacidad de los partidos para aprender de la experiencia. Más concretamente, se trata de saber si van a reeditar la experiencia de los años noventa y dos mil o por el contrario pueden aprender de ella y van a seguir una trayectoria alternativa. Pues en la lista de las causas que nos han llevado a la situación actual de crisis y redefinición de nuestra democracia ocupa un lugar principal la dinámica de polarización puesta en marcha, primero, para liquidar la etapa socialista liderada por Felipe González y, más tarde, para alimentar la capacidad autodestructiva de Aznar en su segunda legislatura. La cuestión que se nos plantea es, por tanto, si los partidos y, especialmente, los nuevos están dispuestos a desarrollar una cultura política distinta, recuperando así los mejores momentos de la transición o si, por el contrario, estamos condenados a repetir lo peor del bipartidismo. En este sentido, hay que tener en cuenta, por lo pronto, que aquella polarización se produjo entre dos partidos políticos que estaban a cuatro casillas de distancia en la escala ideológica (el PSOE de Zapatero estaba en la casilla 4 y el PP estaba en la 8). En tanto que la radicalización de las demandas sociales surgidas con la crisis y la pulsión rupturista de Podemos han colocado a este partido en la casilla 2, nada menos que a seis casillas de distancia del PP (recordemos que la escala izquierda-derecha tiene diez casillas, del 1 al 10).

Con estas premisas, caben dos posibilidades, según que sean los polos, es decir los partidos colocados en los extremos de la escala (Podemos y PP) los que lleven la iniciativa o que ésta recaiga, por el contrario, en las bisagras: PSOE y Ciudadanos. De darse el primer caso, volveríamos a las estrategias de polarización que dominaron la década pasada, las cuales tendrían ahora un efecto de bloqueo, dado el equilibrio de fuerzas entre ambos bloques ideológicos (izquierda y derecha). Pero cabe también la posibilidad de que la iniciativa recaiga sobre las bisagras y que sean éstas las que pongan en marcha un juego alternativo que podemos llamar triangulación, un juego transversal desde el punto de vista ideológico del que ya tuvimos un primer ejemplo en la investidura fallida de Pedro Sánchez, en la que este candidato consiguió 131 votos afirmativos, convirtiéndose en la minoría mayoritaria del Congreso, por delante de la fuerza más votada (PP). El problema de aquella experiencia fue que Podemos no secundó la negociación, haciendo necesaria la repetición de elecciones, algo que nunca había ocurrido en la democracia española y que no suele ocurrir en las democracias del entorno europeo. Si nos detenemos en lo ocurrido entonces, podemos observar que la estrategia de Podemos se concretó en un doble movimiento: en un primer momento, Podemos promovió una maniobra típica de exclusión con el fin de impedir un acuerdo transversal con C's (una «segunda transición» en los términos ya apuntados con anterioridad, que diese prioridad a los temas propios de la «nueva política»y pusiese entre paréntesis los temas ideológicos), para, a conti- 
nuación, promover un acuerdo con IU que diese visibilidad al polo ideológico de la izquierda y presentarlo como la única posibilidad de desbancar al PP. De esta manera, Podemos sacrificó la «nueva política» como posible eje de competición partidista para reeditar la dinámica de polarización de la década pasada, a fin de antagonizar con el PP y presentarlo como representante indiscutible del bloque de las derechas.

Llegados a este punto, conviene recordar el escenario que Giovanni Sartori consideraba más probable en un sistema de partidos de estas características, toda vez que la propia dinámica de polarización conduce de manera más o menos inevitable al debilitamiento del centro como objeto de deseo de la contienda electoral, así como a la aparición de comportamientos anti-sistema, pues la dificultad de encontrar coaliciones viables de gobierno empuja a los partidos al ejercicio de una oposición irresponsable. En cualquier caso, sea cual sea el partido que quede fuera de la coalición dominante es inevitable que el tipo de oposición que ejerza esté marcado por estrategias de deslegitimación del gobierno que se forme ${ }^{13}$.

\section{RECAPITULACIÓN}

He comenzado interpretando la crisis político-institucional del periodo 2010-2015 como una crisis de representación que afecta a la democracia de partidos que se configuró al amparo de la Constitución de 1978. Dicha crisis afecta, en particular, a dos dimensiones del orden institucional: la relación entre representantes y representados y el patrón de opinión pública, de ahí todo el caudaloso debate público sobre la reforma del sistema electoral, la generalización de las primarias, el papel de los servicios públicos de radio y televisión, el formato de los debates electorales, amén de la lucha contra la corrupción y la regeneración de las instituciones. Dada la magnitud y el alcance de los temas en cuestión, la coyuntura pedía una especie de «segunda transición» en virtud de la cual los partidos del cambio fueran capaces de aparcar por un momento sus diferencias ideológicas a fin de abordar el abanico de temas que componen la agenda de la «nueva política». El problema es que, en lugar de apostar por la transversalidad, Podemos optó por reeditar las estrategias de polarización que tan desastrosas consecuencias tuvieron en la crisis del bipartidismo, conduciendo el nuevo pluralismo a una tensión sin precedentes. No deja de resultar paradójico que el paso de la dictadura a la democracia fuese posible mediante dosis

13 SARTORI, Giovanni (1994): Partidos y sistemas de partidos (segunda edición ampliada), Madrid: Alianza Universidad, p. 173. 
muy estimables de colaboración y consenso a nivel de las elites, en tanto que las nuevas elites son incapaces de reformar una partitocracia en crisis, aquejada de altos niveles de corrupción y bajos niveles de calidad democrática. Parece claro que una cosa es criticar la transición y otra bien distinta es sacar de ella las debidas enseñanzas.

Así las cosas, ante la inevitable pregunta ¿qué hay de nuevo en la «nueva política»? no podemos decir — tal como nos gustaría— que la «nueva política» haya sido útil para erradicar la corrupción, promover la regeneración institucional o sanear el sistema político: lamentablemente, la política española sigue discurriendo por los cauces habituales de mediocridad institucional y frentismo estéril que caracterizaron los últimos tiempos del bipartidismo. Lo único que podemos decir es que se ha producido un cambio de paradigma en la relación entre medios y partidos en virtud del cual los medios aprovechan la crisis de representación y la consiguiente debilidad de los viejos partidos para conseguir más control sobre la clase política en su conjunto, en tanto que los nuevos partidos aprovechan las oportunidades ofrecidas por los medios para explorar todas las posibilidades de una democracia mediatizada, ya sea mediante la sustitución del debate político por el ritual de las tertulias o la conversión del parlamento en un plató, de tal suerte que los medios se reservan el derecho a decidir sobre lo que es motivo legítimo de debate y negociación.

Llegados a este punto, caben dos posibilidades: que estemos, efectivamente, en un proceso de transición hacia una democracia de audiencia (tal como suponía Bernard Manin) que incluya el desalineamiento político de los medios, empezando por los públicos, y que facilite, por ende, que los medios velen más por los intereses de la audiencia que por los de sus partidos afines, o que, por el contrario, hayamos iniciado el tránsito hacia alguna suerte de mediocracia en virtud de la cual el deseo compulsivo de obtener presencia mediática vacía la política de contenido y la sustituye por una mera representación teatral, dando lugar así a la «pseudopolítica» ${ }^{14}$ o a la «banalización de la democracia» ${ }^{15}$.

\section{REFERENCIAS}

Ansolabehere, Stephen y Shanto Iyengar (1995): Going Negative. How Political Advertisements Shrink \& Polarize the Electorate, New York: The Free Press.

14 Meyer, Thomas (2002): Media Democracy. How the Media Colonize Politics, Cambridge: Polity.

15 MaIR, Peter (2015): Gobernando el vacío. La banalización de la democracia occidental, Madrid: Alianza editorial 
Castro, Carles (2008): Relato electoral de España (1977-2007). Las claves de la alternancia en el poder durante tres décadas de monarquía parlamentaria, Barcelona: Institut de Ciencies Politiques y Socials.

Corrales, Javier (2005): "In Search of a Theory of Polarization: Lessons from Venezuela, 1999-2005», Revista Europea de Estudios Latinoamericanos y del Caribe - (2011): "Why Polarize? Advantages and Disadvantages of a RationalChoice Analysis of Government-Opposition Relations under Hugo Chávez», en Ponniah y Eastwood: The Revolution in Venezuela. Social and Political Change under Chávez, Harvard University Press.

Fundación BBVA-Ivie, (2015): «Servicios públicos, diferencias territoriales e igualdad de oportunidades».

GonZÁlez, J. J. y P. Chavero (2012): «Política en portada: la construcción mediática de la crisis», en Colino y Cotarelo: España en crisis. Balance de la segunda legislatura de Rodríguez Zapatero, Valencia: Tirant Humanidades: 290-310.

MAIr, Peter (2015): Gobernando el vacío. La banalización de la democracia occidental, Madrid: Alianza editorial.

Manin, Bernard (1998): Los principios del gobierno representativo, Madrid: Alianza Editorial, Ciencias Sociales.

Maravall, J. M. (1984): La política de la transición, Madrid: Taurus.

Meyer, Thomas (2002): Media Democracy. How the Media Colonize Politics, Cambridge: Polity.

SARTORI, Giovanni (1994): Partidos y sistemas de partidos (segunda edición ampliada), Madrid: Alianza Universidad.

Title:

Party Democracy's Crisis and Second Transition.

Summary:

1. Old and new politics. 2. The genealogy of the crisis. 3. The electoral impact of the crisis: the autonomic and general elections of 2015. 4. The new system of parties: limited transition to pluralism polarized pluralism. 5. Summary.

\section{Resumen}

Este artículo comienza presentando la idea de Bernard Manin sobre la evolución de las formas de representación política y el paso de la democracia de partidos a la democracia de audiencia. A continuación, 
hace repaso de la génesis y evolución de la crisis económica con el fin de subrayar algunas de sus consecuencias en el terreno políticoinstitucional. En tercer lugar, se detiene en el análisis del impacto electoral de la crisis de representación en las elecciones autonómicas y generales de 2015, para llegar, por último, a evaluar el nuevo sistema de partidos y sus implicaciones en materia de polarización política.

\begin{abstract}
:
This article begins by presenting the Bernard Manin's argument on the evolution of the political representation and the transition from party democracy to audience democracy. It continues by taking into account the origins and evolution of the economic crisis in order to underline some of its consequences concerning the political and institutional arena. Third, it analyzes the electoral impact of the crisis of representation on the regional and general 2015 elections. Finally, the article evaluates the new party system and its implications with regard to the issue of political polarization.
\end{abstract}

\title{
Palabras clave:
}

Democracia de partidos, democracia de audiencia, crisis de representación, sistema de partidos y pluralismo polarizado.

\section{Key words:}

Party democracy, audience democracy, crisis of representation, party system, and polarized pluralism. 